\title{
MOS guidance using a neural network for the rainfall forecast over India
}

\author{
Ashok Kumar ${ }^{1, *}$, Ch Sridevi ${ }^{1}$, V R Durai ${ }^{1}, \mathrm{~K} \mathrm{~K} \mathrm{Singh}^{1}$, \\ P MukhopadhyaY ${ }^{2}$ and N Chattopadhyay ${ }^{3}$ \\ ${ }^{1}$ India Meteorological Department, New Delhi 110 003, India. \\ ${ }^{2}$ India Institute of Tropical Meteorology, Pune 411 017, India. \\ ${ }^{3}$ India Meteorological Department, Pune 411 005, India. \\ *Corresponding author.e-mail: ashokscg@gmail.com
}

MS received 16 April 2018; revised 20 August 2018; accepted 26 August 2018; published online 13 May 2019

In the present study, a model output statistics (MOS) guidance model was developed by using the neural network technique for a bias-corrected rainfall forecast. The model was developed over the Indian window $\left(0-40^{\circ} \mathrm{N}\right.$ and $\left.60-100^{\circ} \mathrm{E}\right)$ by using the observed and global forecast system (GFS) T-1534 model output (up to 5 days) at a $0.125^{\circ} \times 0.125^{\circ}$ regular grid during the summer monsoon (June-September) 2016 . The skill of the developed MOS model forecast against the observed $0.125^{\circ} \times 0.125^{\circ}$ grid rainfall data is obtained for the summer monsoon (June-September) 2017. The skill of the MOS model rainfall forecast is found to show good improvement over the T-1534 model's direct forecast over the Indian window. In general, the T-1534 model's direct forecast shows high skill but the forecast obtained by using the MOS model shows better skill than the direct model's forecast, although a major improvement is seen for the Day 1 forecast at the national level. So the skill of the bias-corrected rainfall forecast by using the MOS guidance and the T-1534 model output is high and has the potential of being used as an operational forecast over the Indian region.

Keywords. MOS; neural network; T-1534; regular grid; monsoon season; Indian window.

\section{Introduction}

The work on the statistical interpretation (SI) (perfect prognosis method $(\mathrm{PPM}) /$ model output statistics (MOS)) of models was started in 1990 after the installation of the first general circulation model (GCM) R-40, at the National Centre for Medium Range Weather Forecasting (NCMRWF) in 1989. The SI forecast was started and is used for the preparation of the final forecast for different locations and it was started for five stations for the first time in the monsoon of 1991. Later on, in the monsoon of 1994, the T-80 model-based SI forecast was started, as the T-80 model was installed in 1993. The SI forecast for the winter season was started in 1995-1996. Later on, it was developed for more number of stations and for higher resolution models such as the T-254 and T-574 models as well. For all these location-specific models, the PPM technique was used and predictors are selected for each location separately by using the stepwise regression procedure (Kumar and Maini 1996).

In the present study, the SI technique was attempted with the MOS guidance for rainfall forecast at a $0.125^{\circ} \times 0.125^{\circ}$ grid over the Indian window $\left(0-40^{\circ} \mathrm{N}\right.$ and $\left.60-100^{\circ} \mathrm{E}\right)$. The predictors 
used for developing the MOS guidance equations are based upon the knowledge of the most important and frequently selected predictors from the earlier work mentioned above. The weather parameters used as predictors in the present study are mentioned in the following sections. The predictand is the observed rainfall at all grids for the considered Indian window. The neural network technique (Jain et al. 2002) is used for developing the MOS equations at all grids of the abovementioned Indian window up to 5 days.

\section{Data}

The observational rainfall data which is used as the predictand is at a resolution of $12.5 \mathrm{~km}$ over the Indian window $\left(0-40^{\circ} \mathrm{N}\right.$ and $\left.60-100^{\circ} \mathrm{E}\right)$ prepared by downscaling the $25 \mathrm{~km}$ gridded rainfall obtained from India Meteorological Department (IMD), Pune.

The forecasted weather parameters which are used as the predictors are also obtained for the Indian window at a $0.125^{\circ} \times 0.125^{\circ}$ grid resolution from the global forecast system (GFS) T-1534 model post-processed output. Six weather parameters are used as the predictors, i.e., forecasted rainfall, cloud amount, mean relative humidity (1000-500 hPa), $850 \mathrm{hPa}$ vorticity, $1000 \mathrm{hPa}$ zonal wind and $500 \mathrm{hPa}$ vertical velocity. These six weather parameters explain most of the variance in the observed rainfall (Glahn and Lowry 1972; Maini et al. 2002). The forecast data of these parameters is prepared from three hourly postprocessed output based upon the 0 GMT initial condition run of the T-1534 model up to 5 days.

All these forecasts are obtained from the GFS model i.e. run at the IMD, New Delhi. The GFS is a primitive equation spectral global model with the state-of-the-art dynamics and physics (Kanamitsu 1989; Kalnay and Baker 1990; Kanamitsu et al. 1991; Durai et al. 2010; Durai and RoyBhowmik 2013). For the GFS (gsm.v12.0.2) adopted from the National Centre for Environmental Prediction (NCEP), at T1534L64 ( 12 km), in the horizontal and the vertical resolutions, there are 64 hybrid sigma-pressure (Sela 2009) layers with the top layer centred around $0.27 \mathrm{hPa}(\sim 55 \mathrm{~km})$ that has been operational on ADITYA High Performance Computing Systems (HPCS) at the Indian Institute of Tropical Meteorology (IITM), Pune, twice a day (00 and 12 UTC) since 1 December 2016 to give a deterministic forecast in the short to the medium range. The initial conditions for the GFS model are generated from the NCEP-based global three-dimensional variational (3D-VAR) grid point statistical interpolation (GSI 3.0.0) scheme until 30 June 2016. Since 1 July 2016, the initial conditions have been generated from the NCEP-based ensemble Kalman filter (EnKF) component of the hybrid global data assimilation system (GDAS) run on BHASKARA HPCS at the NCMRWF.

The current operational dynamical core of the GFS/GSM is based on the two time-level semiimplicit semi-Lagrangian discretisation (Sela 2009, 2010) with the 3D Hermite interpolation (the dynamical core still supporting the three time-level Eulerian approach). The semi-Lagrangian advection calculations and the treatment of physics are done on a linear, reduced (for computational economy) Gaussian grid in the horizontal domain. The semi-implicit treatment and implicit eighth-order horizontal diffusion are performed in the spectral space. This requires the application of Fourier and Legendre transforms to convert between spectral and grid-point spaces. To improve the accuracy of the associated Legendre function computation at higher wave numbers, an extended range arithmetic (Juang 2014) is used. Also, a correction is applied to the global mean ozone to account for

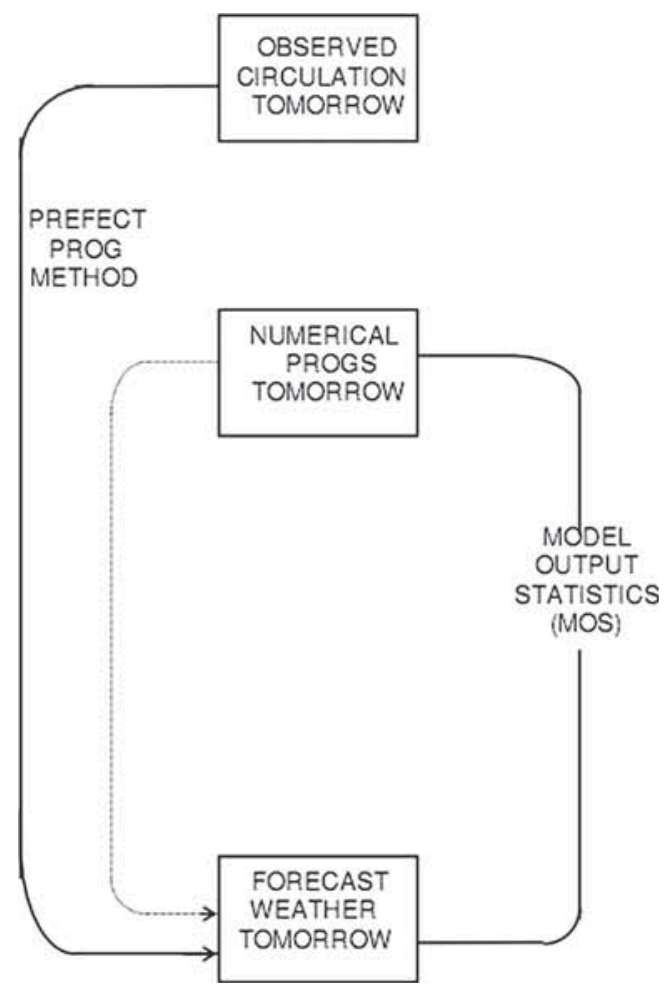

Figure 1. Two methods of SI weather forecasting in the schematic form. 
the non-conservation during the semi-Lagrangian advection step. For the three time-level Eulerian option of dynamics, a positive-definite tracer transport formulation (Yang 2009) is used in the vertical.

It is important to note that the same physical parameterisation package is used across all horizontal and vertical resolutions. Upgrades to the physical parameterisations are ongoing and occur on the average of every other year. The model is based on the usual expressions of conservation of mass, momentum, energy and moisture at T1534 spectral truncation, approximately a $13-\mathrm{km}$ physics (Gaussian) grid and 64 levels in a hybrid sigma pressure vertical coordinate. Output is posted on a $0.125^{\circ}$ equally spaced longitude/latitude grid with a 3-243-hr forecast interval. Details about the GFS are available at http://www.emc.ncep.noaa.gov/ GFS/doc.php.
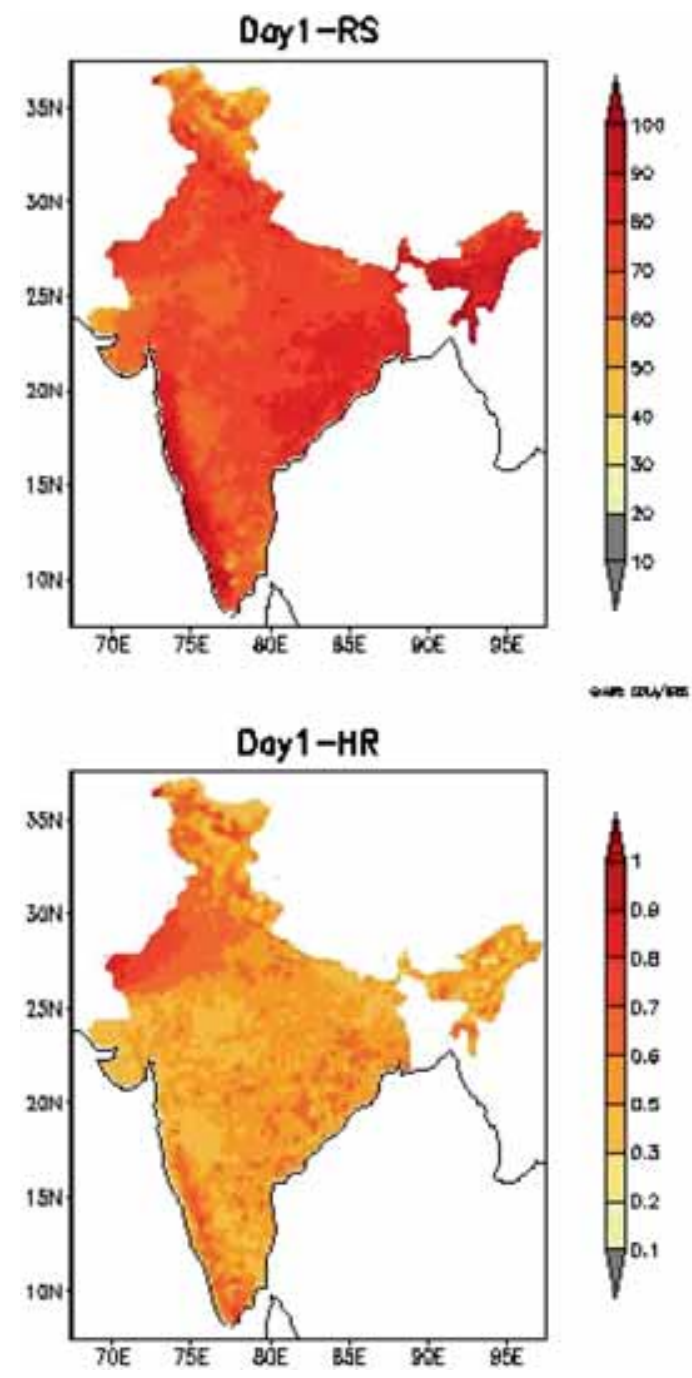

\section{Methodology}

\subsection{MOS guidance approach}

The approach used for MOS guidance models is one of the techniques used for the SI PPM models/MOS forecast which is applied for getting the bias-free forecast from the NWP models forecast for different weather parameters. Surface weather elements such as rainfall, maximum temperature and minimum temperature are highly dependent on local topographic and environmental conditions. As in numerical weather prediction (NWP) models and particularly in the GCM, it is very difficult to include each and every aspect of these local conditions at all locations; hence, the direct model output (DMO) forecast obtained from the NWP models may have problems. But upper air circulation at a specific location is not so dependent
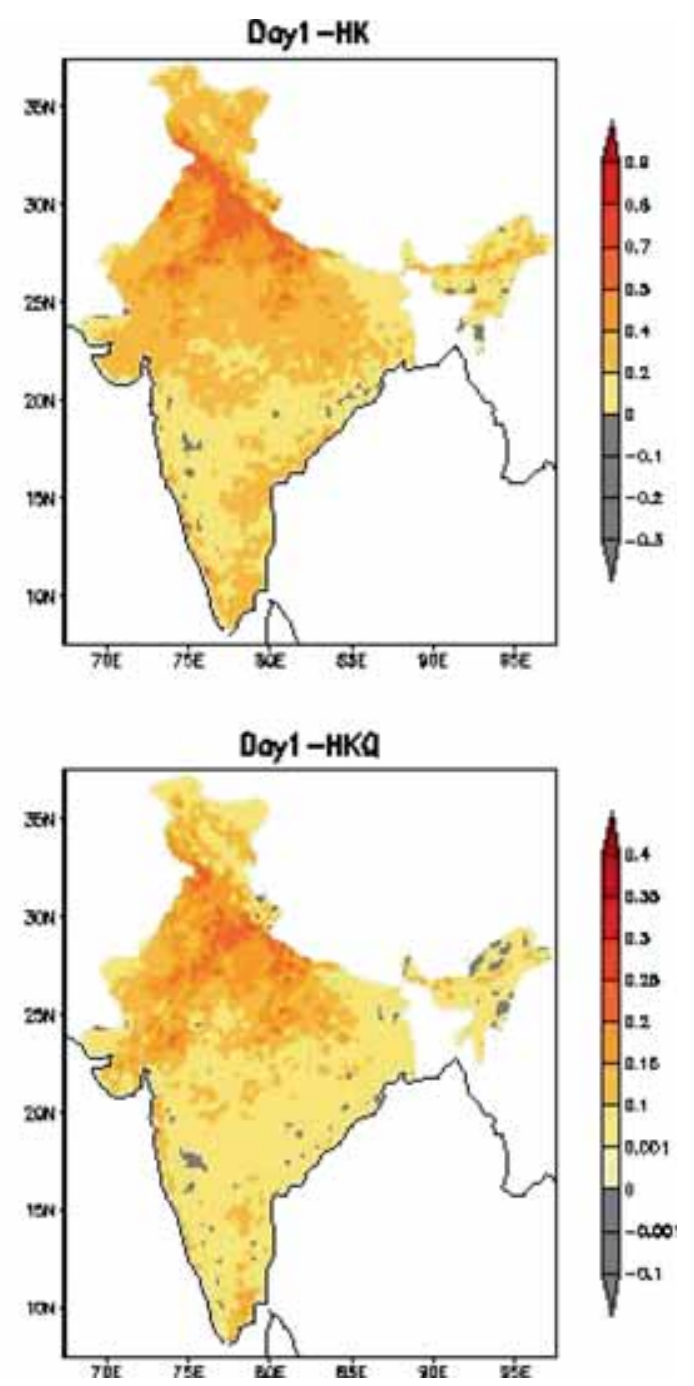

Figure 2. Skill scores of the GFS T1534 Day 1 direct rainfall forecast over the Indian region during the summer monsoon (JJAS) 2017. 
on local conditions and can be obtained from the analysis or forecast of a GCM easily. A statistical relation developed between the upper air circulation around the location of interest and observed values of the surface weather element at the location will definitely account for the effect of these local conditions. This indicates that the SI forecast so obtained will have better skill as compared to the DMO forecast (Kumar and Maini 1996). Basically, two methods are used for the SI forecast, i.e., PPM and MOS (figure 1).

In the PPM approach (Maini et al. 2002; Bhardwaj et al. 2009), a statistical relation is derived that relates a large sample of observed surface elements (predictands) to concurrent observed surface and upper air reports, i.e., analysis (predictors). In order to get a forecast for the appropriate valid time, values of the predictors obtained from the NWP models are substituted in the relation developed. This approach assumes that the model forecasts are 'perfect'. Hence, this approach has a disadvantage that it does not account for the systematic biases and errors of the model. This problem can be solved by using the unbiased model forecast, which can be obtained just on the basis of model analysis and forecast data for the previous 1 or 2 months. A major advantage of this method is that stable forecasting relations can be derived from a long period of record, so its forecast improves as the NWP model forecast improves.

In the MOS approach, a statistical relation is developed using observed surface weather elements (predictands) and the NWP model forecasts for upper air circulation variables (predictors). As such, a separate MOS relation is developed for a particular day forecast. To make operational
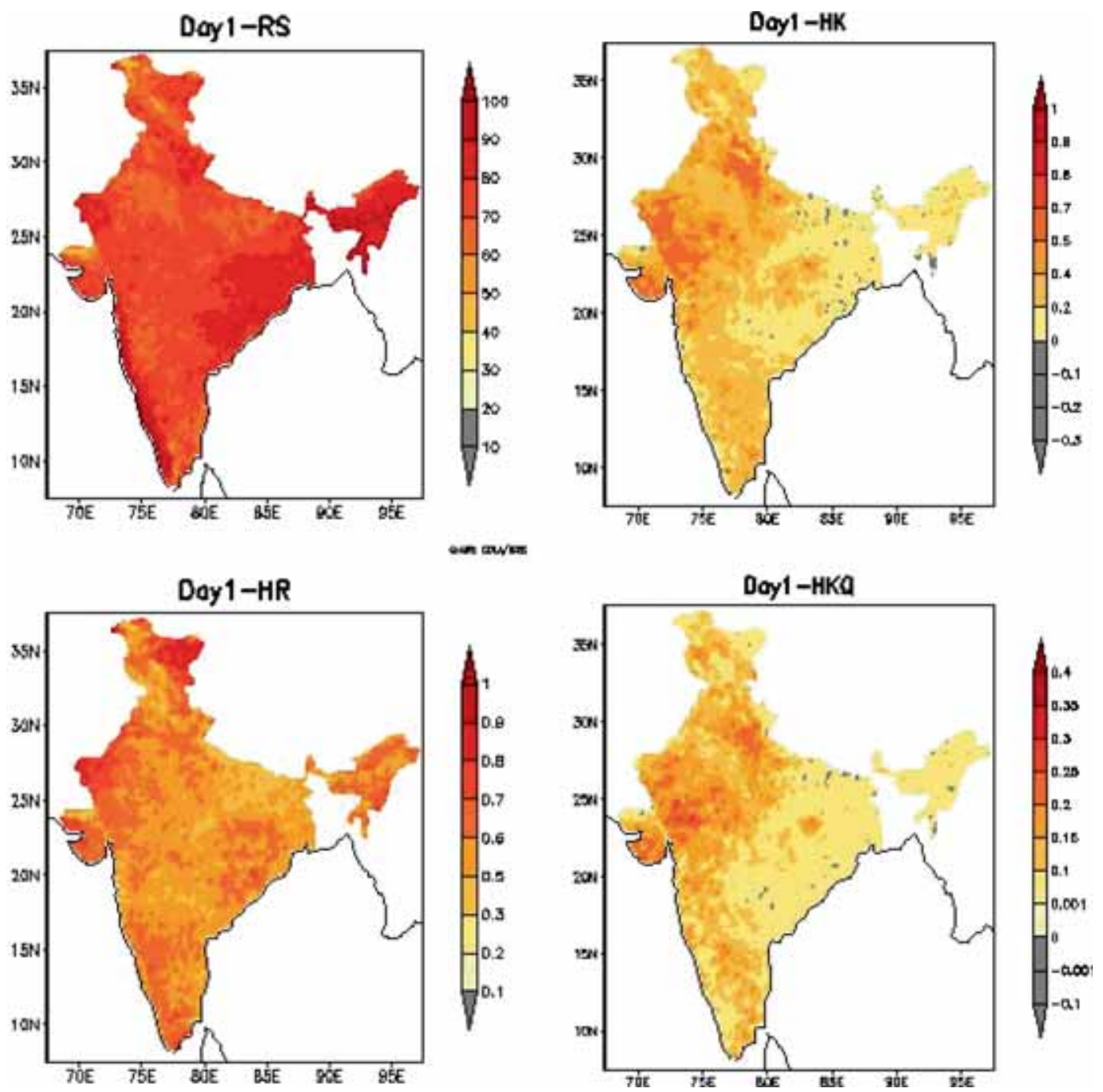

Figure 3. Skill scores for GFS T1534 MOS guidance Day 1 rainfall forecast over the Indian region during summer monsoon (JJAS) 2017. 
forecasts, MOS relations are usually applied to the same dynamical model that provided the developmental sample. It has an advantage that MOS relations account for some of the biases and systematic errors found in the NWP model. A disadvantage of this technique is that the model output data for a sufficiently longer period is required so as to derive a stable relation, but this disadvantage can be overcome by using the neural network technique. Hence, it cannot be applied immediately when a new NWP model is made operational, and also if the NWP model undergoes a major change, the MOS relations will have to be developed again.

The MOS guidance models for rainfall are developed by using the neural network technique explained in the following section, for a 5-day forecast. The predictand used for developing these MOS guidance equations is the observed gridded rainfall data at $0.125^{\circ} \times 0.125^{\circ}$ over the Indian window. But the knowledge for using the most important and frequently selected weather parameters as predictors is used from the earlier work of developing the PPM models for different locations (Maini et al. 2002; Bhardwaj et al. 2009). As per these models, mean relative humidity (1000$500 \mathrm{hPa}$ ), $850 \mathrm{hPa}$ vorticity and $850 \mathrm{hPa}$ zonal wind are found to be the mostly selected weather parameters for most of the locations. But, in the present study, two basic weather parameters were also considered as predictors, i.e., forecasted rainfall and cloud amount (Glahn and Lowry 1972). After studying the earlier work, it had been found that the $1000-\mathrm{hPa}$ zonal wind could be a more efficient predictor as compared to the $850-\mathrm{hPa}$ zonal wind which is nearer the surface during the monsoon season. It had also been found that
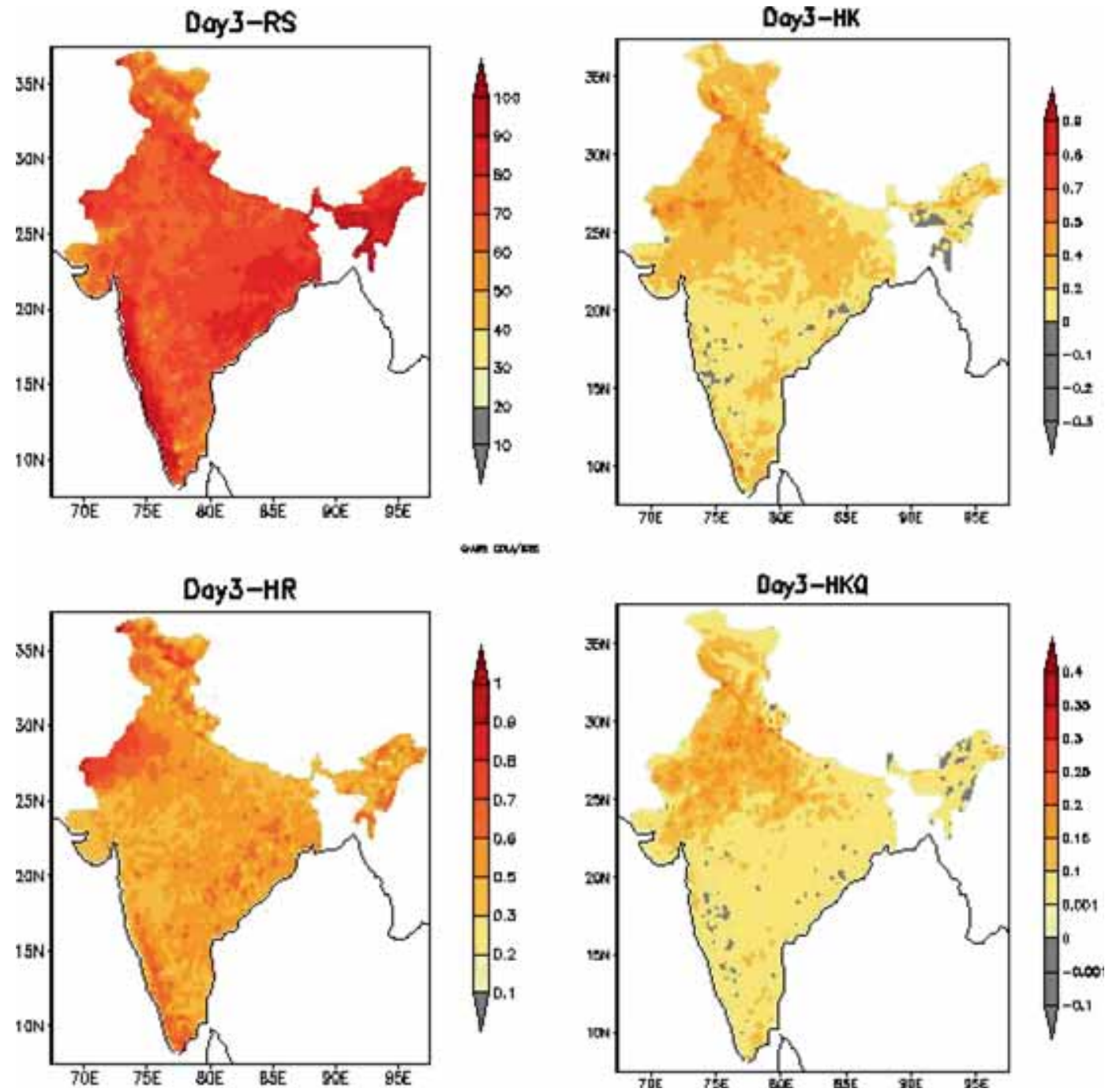

Figure 4. Skill scores for GFS T1534 Day 3 direct rainfall forecast over the Indian region during summer monsoon (JJAS) 2017. 
the $500-\mathrm{hPa}$ vertical velocity is another important weather parameter which can be added to the list of the most important and frequently selected predictors. Hence, we have considered in all six predictors, viz. forecasted rainfall, cloud amount, mean relative humidity $(1000-500 \mathrm{hPa}), 850 \mathrm{hPa}$ vorticity, $1000 \mathrm{hPa}$ zonal wind and $500 \mathrm{hPa}$ vertical velocity.

Although many seasonal synoptic situations are not included in the present study, the dry and wet spells for 122 days are considered and daily rainfall amounts are related with upper air weather parameters and model forecasted rainfall spread over 122 days of monsoon season 2016. Moreover, the relation is developed by using the neural network in which derived patterns of daily rainfall amounts, i.e., the predictand and all the predictors are used, which has better capability of training the model as compared to normal regression. Hence, in this study, the MOS guidance has improved the rainfall forecast, although it is based upon only one season.

\subsection{Neural network technique}

The basic technique used in this study is the neural network. Neural networks have a massively parallel, layered structure with each layer consisting of several nodes called neurons. They provide a mapping from the input vector $x i, i=1,2, \ldots, n$ to the output vector $y j, j=1,2, \ldots, m$. Besides the input and output layers, the network contains one or more hidden layers.

Each neuron produces an output $O=f(Z)$, where $Z=z i w i+b, z i(i=1,2, \ldots, n)$ is the input to the given neuron, $f(Z)$ is the activation function and is usually taken to be the sigmoidal function $1 /[1+\exp (-Z)]$, wi is the weight associated with the network and $b$ is the bias of the neuron. The
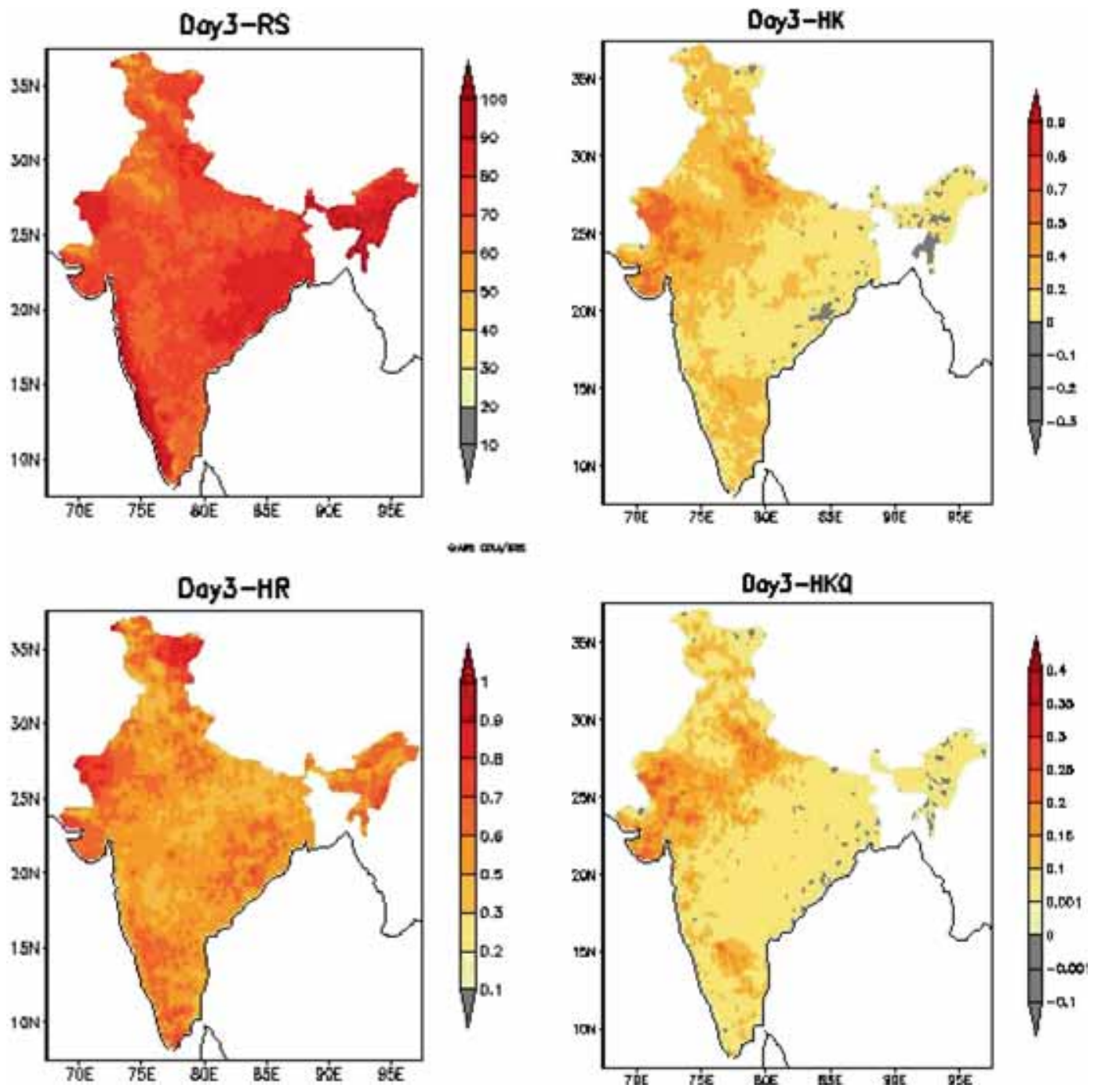

Figure 5. Skill scores for GFS T1534 MOS guidance Day 3 rainfall forecast over the Indian region during summer monsoon (JJAS) 2017. 
weight $w i$ and the bias $b$ represent the parameters of the network which are to be determined by using the training data set of the pattern to be learned (Pankaj et al. 2002). More details on neural networks and their applications can be found in text books on neural networks (Kumar et al. 2012a, b).

The training algorithm used in the neural network for the minimisation of error is the procedure of conjugate gradients complemented by simulated annealing to evade local minima. The method of conjugate gradients is expected to be more efficient than the more commonly used back propagation algorithm, and hence, the network is expected to learn faster. The simulated annealing method is necessary to escape from the local minima which are usually present abundantly in the error function. The error measure was taken as the usual mean squared sum of errors.
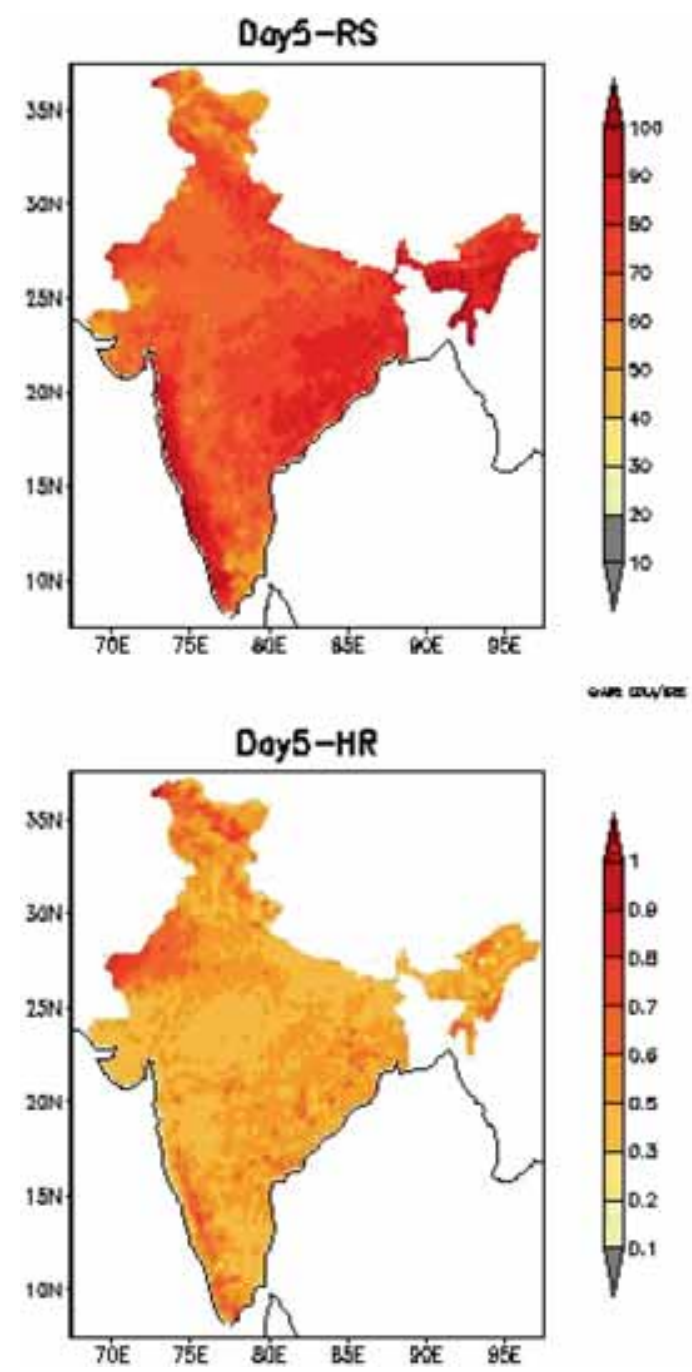

The cubic root of precipitation is computed before applying the neural network technique to the observed and the forecast rainfall data obtained from the four models. This has reduced the highly variable rainfall data to data having a near normal distribution of a lesser variability. The maximum and minimum are then obtained for each series and the data is transformed to have values between 0.1 and 0.9 as per the following formulations.

$$
x_{\bmod }=0.1+0.8 \times\left(x-x_{\min }\right) /\left(x_{\max }-x_{\min }\right) .
$$

The observed rainfall patterns are then considered as the output layer and the forecasted weather parameter patterns used as six predictors are taken as the input layer. A neural network with one
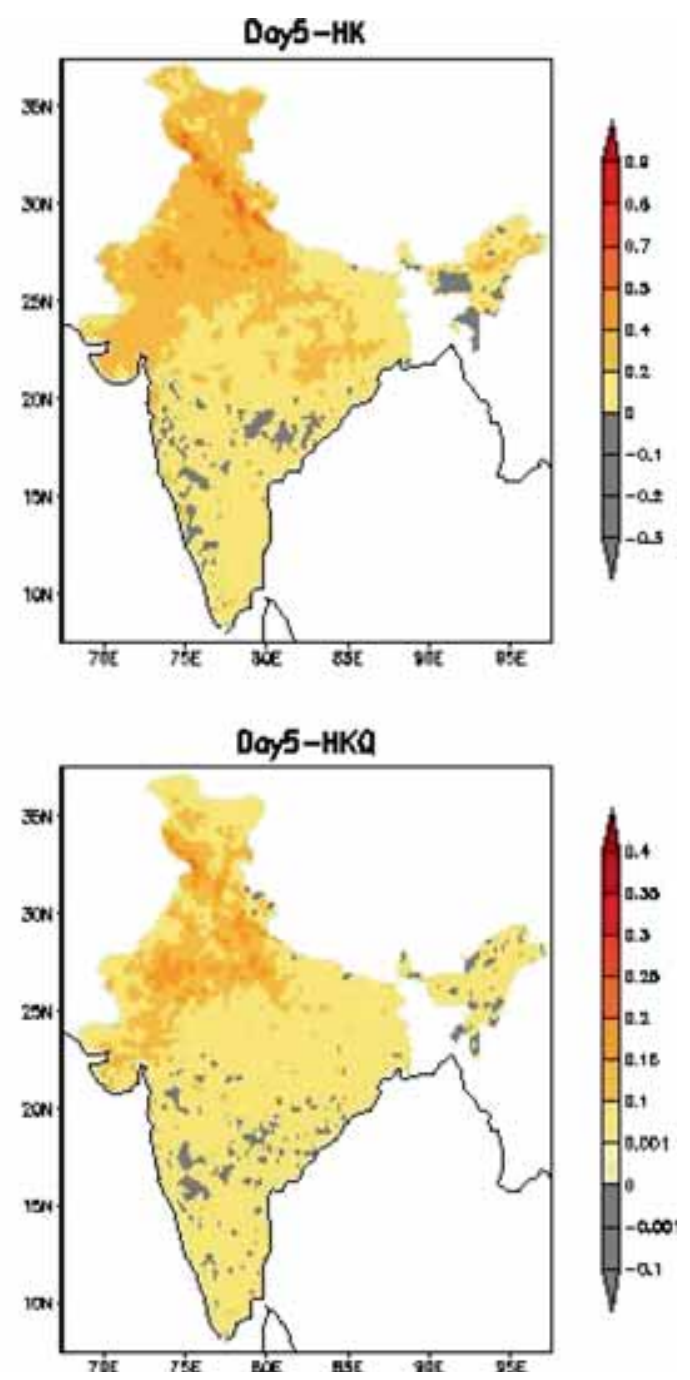

Figure 6. Skill scores for GFS T1534 Day 5 direct rainfall forecast over the Indian region during summer monsoon (JJAS) 2017. 

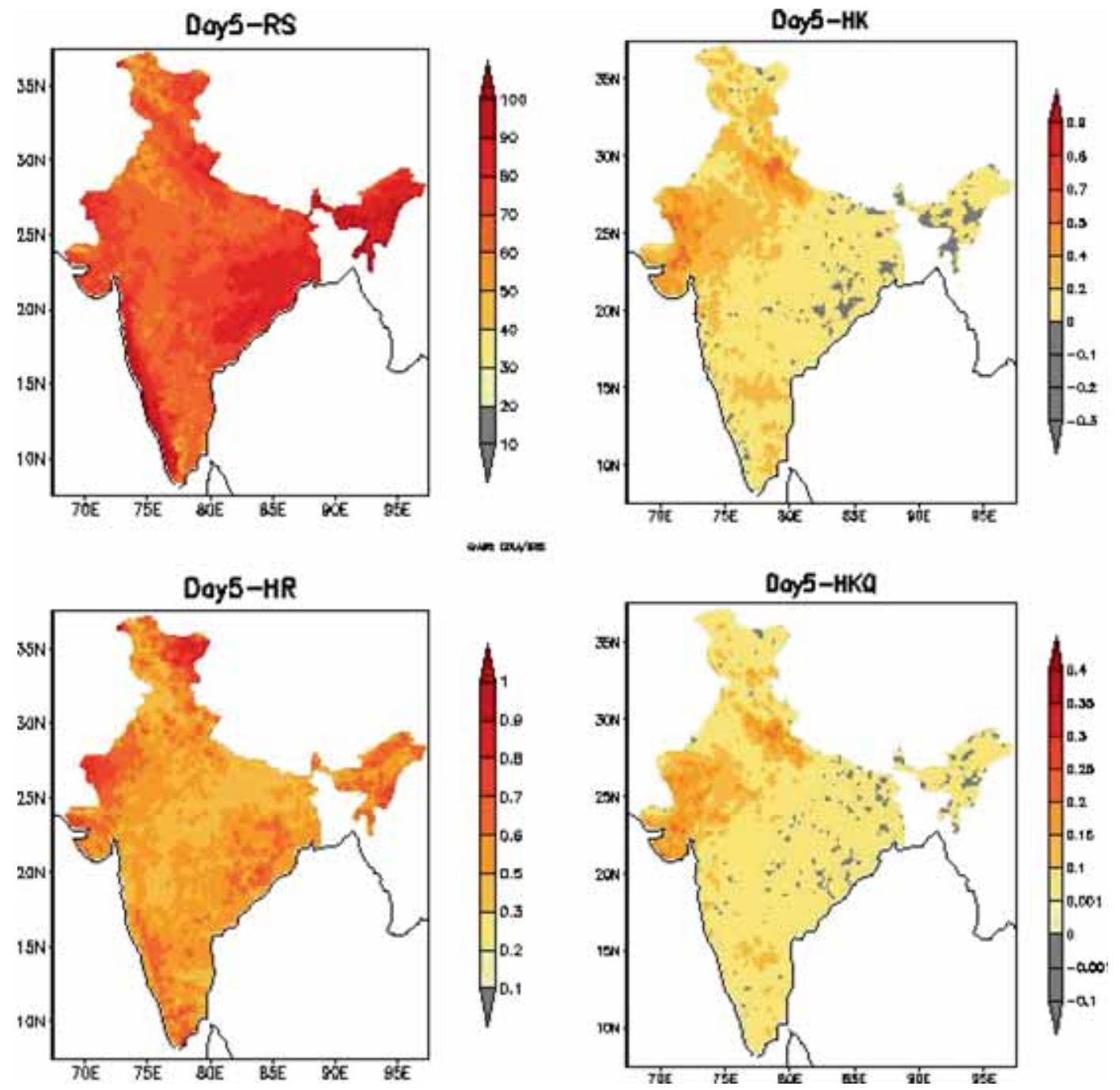

Figure 7. Skill scores of the GFS T1534 MOS guidance Day 5 rainfall forecast over the Indian region during the summer monsoon (JJAS) 2017.

hidden layer and three neurons is used to train the network and develop the relation between the observed rainfall and the four forecasts obtained from four different models. A simple sigmoidal function, $1 /[1+\exp (-Z)]$, is used as the activation function.

\subsection{Skill scores used for verification study}

The standard skill scores are used for yes/no and quantitative rainfall forecast verification (Murphy and Katz 1985). Ratio score (RS) and Hanssen and Kuiper's (HK) scores are used for yes/no rainfall forecast. Hit rate (HR) and HK's score for quantitative rainfall (HKQ) forecast are used. Besides this, the correct, usable and unusable skill scores were also obtained for rainfall amounts. The criterion for this is defined as follows:

\begin{tabular}{llc}
\hline Parameter & Category & \multicolumn{1}{c}{ Error structure } \\
\hline Rainfall & & If observed $\mathrm{r} / \mathrm{f}$ is out by \\
& Correct & diff $\leq 2.5 \mathrm{~mm}$ for $\leq 10 \mathrm{~mm}$ \\
& Usable & $2.5 \mathrm{~mm}<$ diff $\leq 5 \mathrm{~mm}$ for \\
& Unusable & diff $>5 \mathrm{~mm}$ for $\leq 10 \mathrm{~mm}$ \\
\hline
\end{tabular}

\section{Results and discussion}

The observed and forecasted rainfall values (from the GFS T-1534 output) at the regular grid of $0.125^{\circ} \times 0.125^{\circ}$ resolution for the Indian window $0-40^{\circ} \mathrm{N}$ and $60^{\circ}-100^{\circ} \mathrm{E}$ were considered and the skill scores are calculated for the Indian window for the direct model forecast from the T-1534 model output and MOS guidance derived rainfall forecast. 

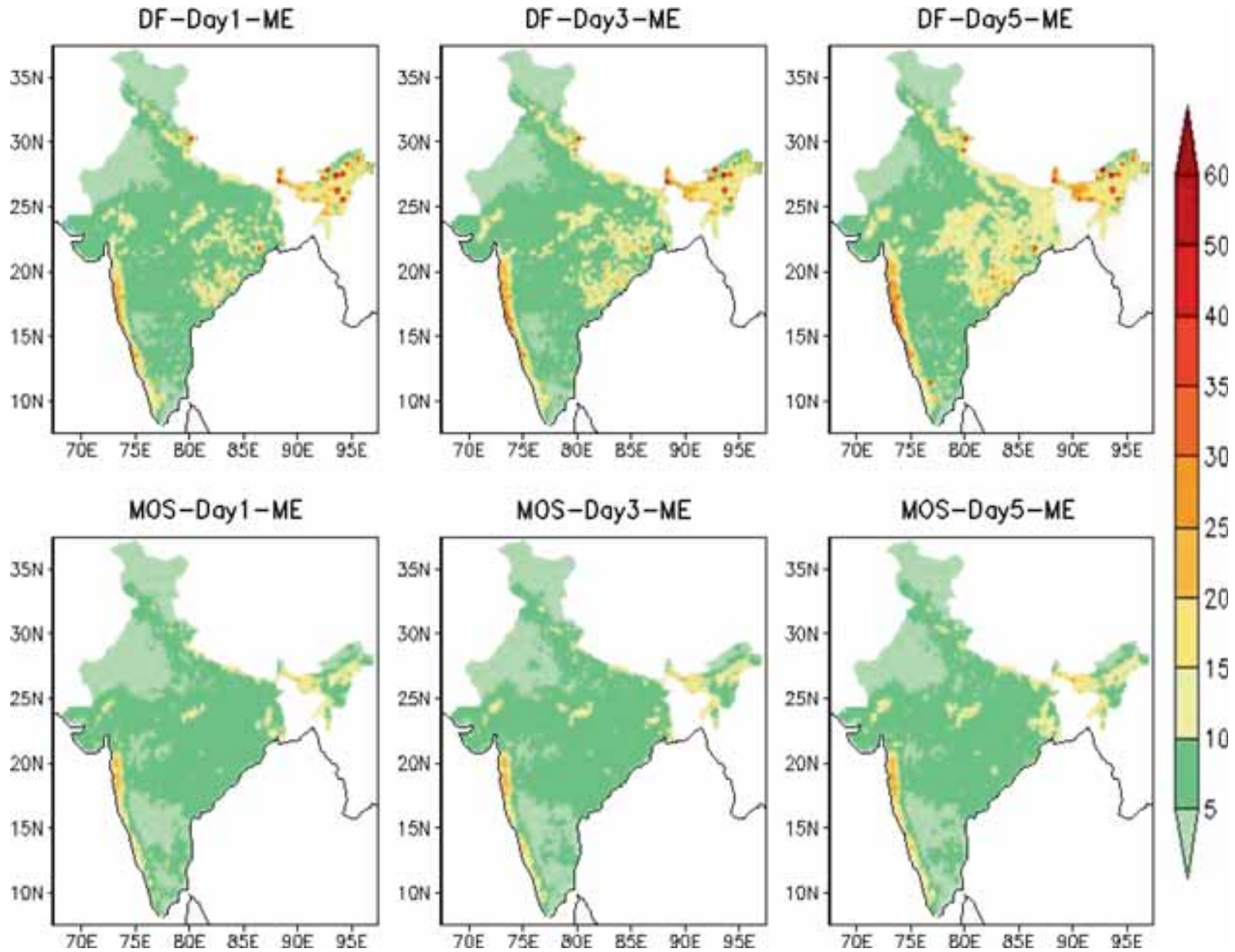

Figure 8. Mean error of the GFS T1534 direct forecast and the MOS guidance rainfall forecast (Day 1, Day 3 and Day 5) over the Indian region during the summer monsoon (JJAS) 2017.
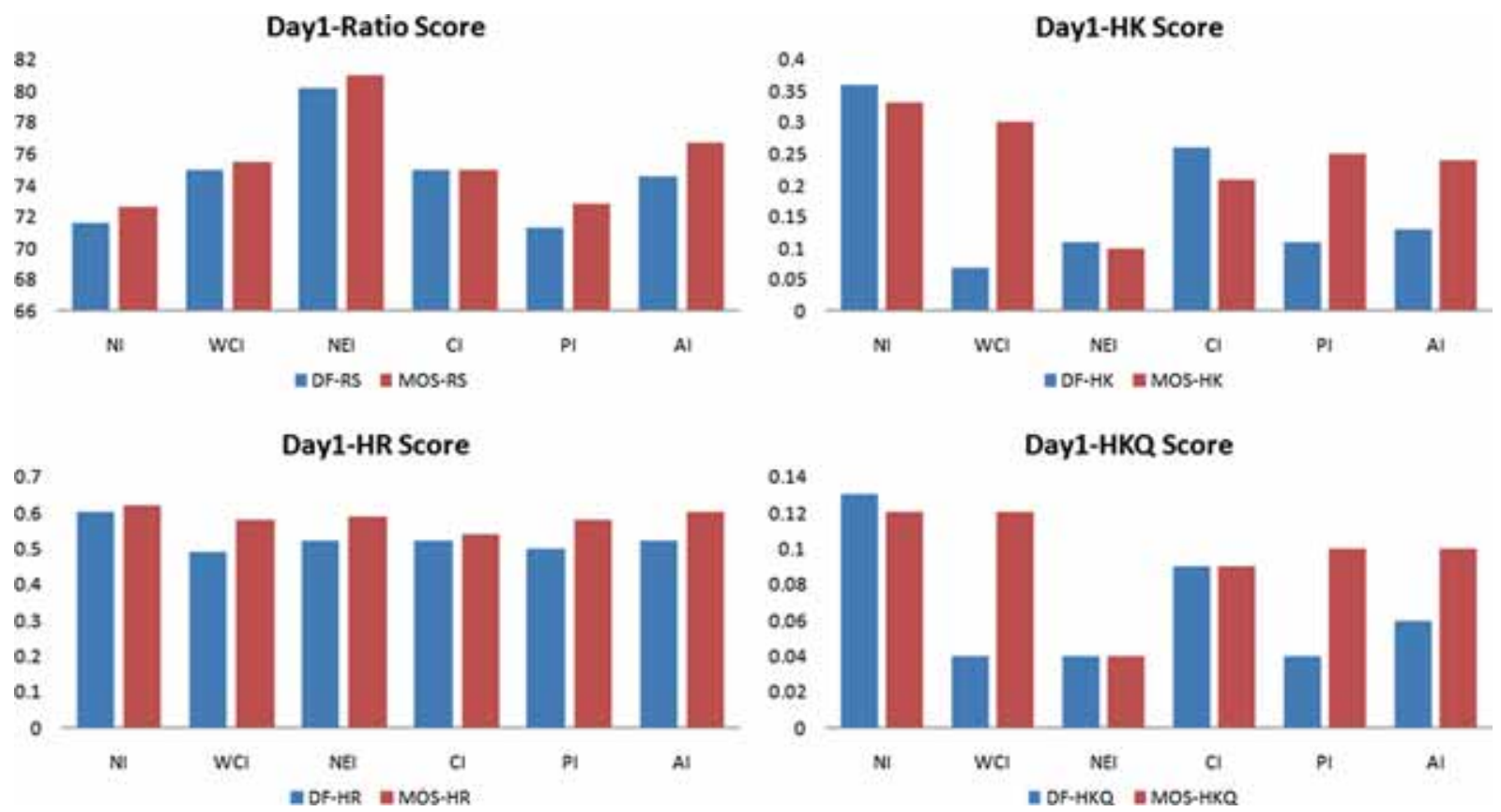

Figure 9. Skill scores (RS, HK, HR and HKQ) of the GFS T1534 direct and the MOS guidance Day 1 rainfall forecast during the summer monsoon (JJAS) 2017 over five homogeneous regions along with all other regions of India. 
Day3-Ratio Score

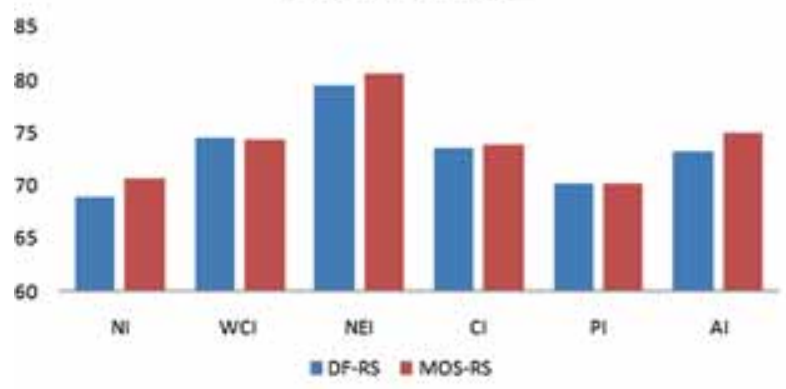

Day3 HR Score

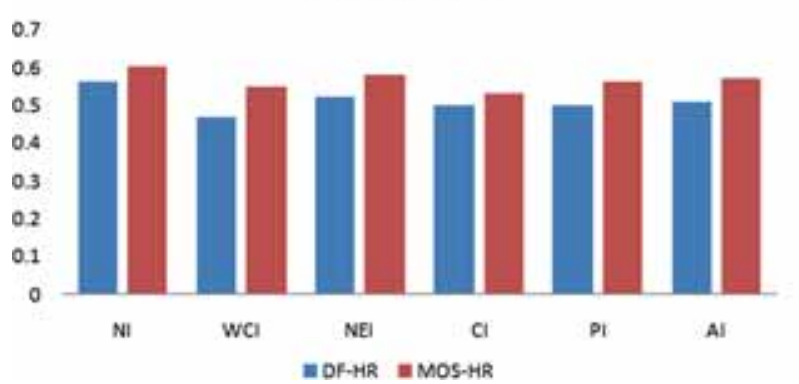

Day3- HK Score

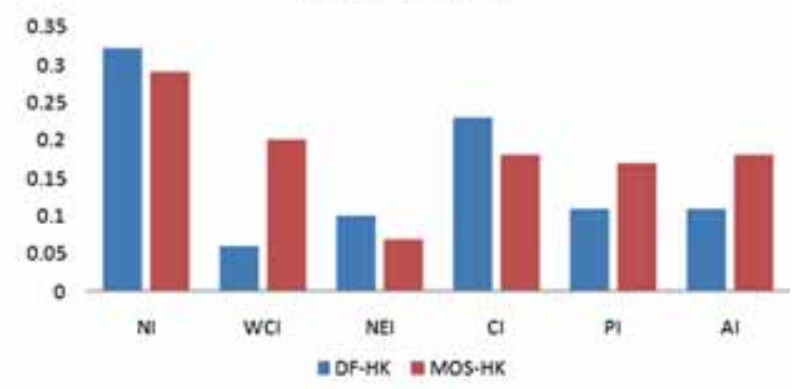

Day3 - HKQ Score

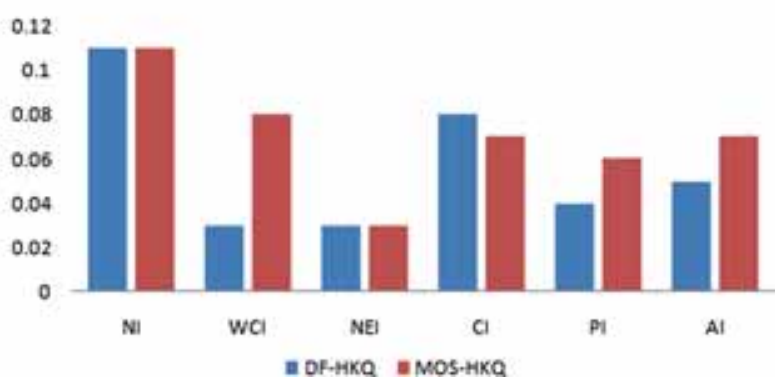

Figure 10. Skill scores (RS, HK, HR and HKQ) of the GFS T1534 direct and the MOS guidance Day 3 rainfall forecast during the summer monsoon (JJAS) 2017 over five homogeneous regions along with all other regions of India.

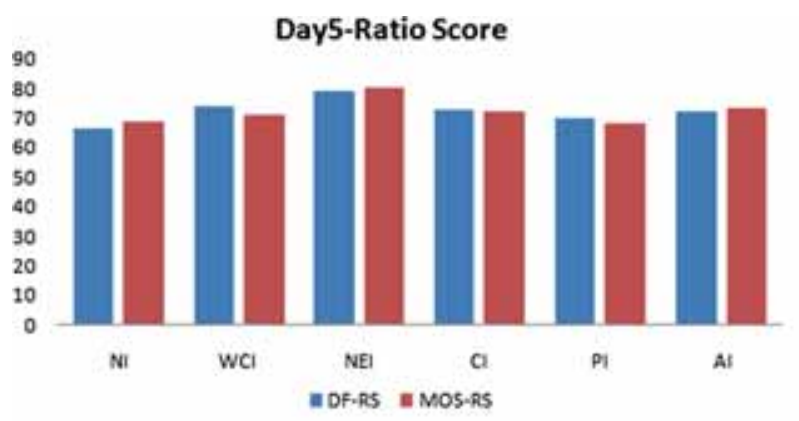

Day5-HR Score

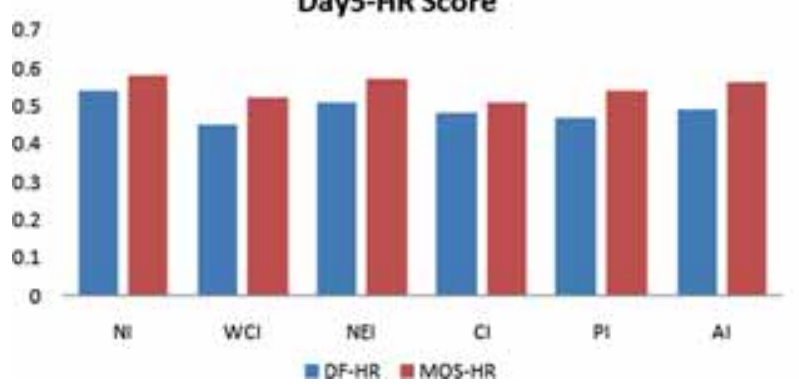

\section{Day5 - HK Score}

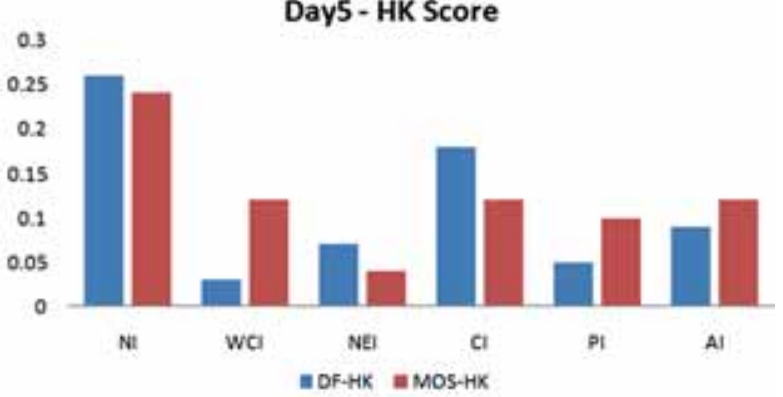

Day5-HKQ Score

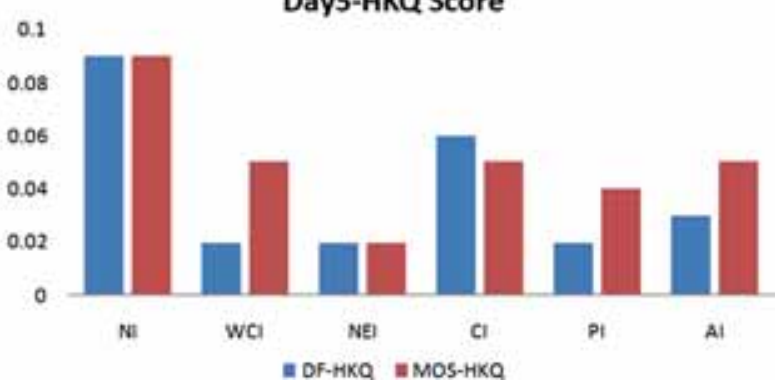

Figure 11. Skill scores (RS, HK, HR and HKQ) of the GFS T1534 direct and the MOS guidance Day 5 rainfall forecast during the summer monsoon (JJAS) 2017 over five homogeneous regions along with all other regions of India.

The skill scores are calculated for the monsoon season (JJAS) 2017. Skill scores RS, HK, HR and HKQ of the GFS direct forecast (DF) are plotted in figures 2-4 and of the GFS MOS guidance are plotted in figures 5-7 for Day 1, Day 3 and Day 5 and the mean absolute error (ME) from both forecasts are plotted in figure 8. All skill scores are improved after applying MOS guidance over most parts of the country for all Day 1, Day 3 and Day 5 forecasts.

A comparative study is conducted for the direct model rainfall forecast and the MOS guidance derived rainfall forecast, for the Indian window by obtaining skill scores for all grids over India 

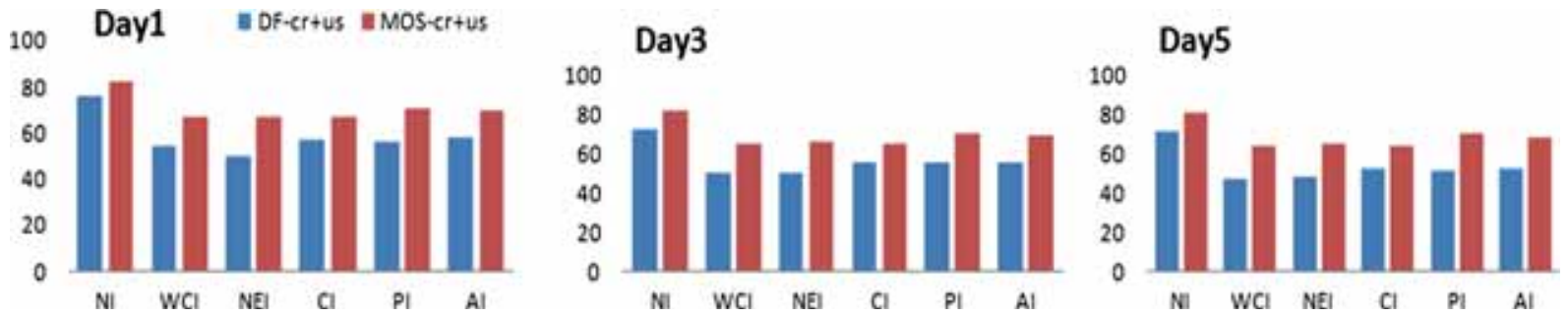

Figure 12. Usable skill score of the GFS T1534 direct and the MOS guidance Day 1, Day 3 and Day 5 rainfall forecast during the summer monsoon 2017 over five homogeneous regions along with all other regions of India.

as a whole $\left(7-37^{\circ} \mathrm{N}\right.$ and $\left.67-100^{\circ} \mathrm{E}\right)$ and over five different homogeneous regions, i.e., North India (NI) $\left(25-35^{\circ} \mathrm{N}\right.$ and $\left.70-85^{\circ} \mathrm{E}\right)$, West Coast of India (WCI) $\left(10-20^{\circ} \mathrm{N}\right.$ and $\left.70-78^{\circ} \mathrm{E}\right)$, North East India (NEI) $\left(22-30^{\circ} \mathrm{N}\right.$ and $\left.85-100^{\circ} \mathrm{E}\right)$, Central India (CI) $\left(22-28^{\circ} \mathrm{N}\right.$ and $\left.73-90^{\circ} \mathrm{E}\right)$ and Peninsular India (PI) $\left(7-21^{\circ} \mathrm{N}\right.$ and $\left.74-85^{\circ} \mathrm{E}\right)$ during the monsoon season 2017 (figures 9-11). The skill of the MOS guidance rainfall forecast is found to be better for overall India for Day 1, Day 3 and Day 5. Over homogeneous regions also, it shows better skill for most of the regions, although for a few regions, the scores are slightly less.

The usability scores for the direct rainfall forecast and MOS guidance derived rainfall forecast are also obtained for all India and five homogeneous regions. These scores are plotted in figure 12 . These scores show up to $20 \%$ improvement for all India and all regions for Day 1, Day 3 and Day 5 after applying MOS guidance.

\section{Conclusions}

As is clear from the results, the skill of the rainfall forecast has improved after applying MOS guidance. It can be observed from the above-mentioned figures that the T-1534 model direct forecast shows high skill and the forecast obtained by using the MOS model shows better skill than the direct forecast. As such, the skill of the bias corrected rainfall forecast by using the MOS guidance and the T-1534 model output is quite high and has the potential of being used as an operational forecast over the Indian region.

As the resolution of the GFS T-1534 model is $12 \mathrm{~km}$, one can derive from the MOS guidance rainfall forecast for the Indian window, the forecast at any level, i.e., district, block, city or for any particular location. The forecast so derived will have the high skill and the potential of being used as an operational forecast throughout the country.

\section{Acknowledgements}

The authors gratefully acknowledge the valuable guidance given by Dr K J Ramesh, DGM, IMD, New Delhi, for carrying out the research work and writing and formatting this paper. Thanks are also due to IMD, Pune, for providing regular grid data for rainfall for the Indian window. Finally, the authors wish to thank the NWP division, IMD, New Delhi, for the assistance provided.

\section{References}

Bhardwaj A, Kumar A and Maini P 2009 Statistical interpretation forecast and its skill during monsoon season in India; Int. J. Meteorol. 34(336) 39-47.

Durai V R and Roy Bhowmik S K 2013 Prediction of Indian summer monsoon in short to medium range time scale with high resolution global forecast system (GFS) T574 and T382; Clim. Dyn. 42(5-6) 1527-1551.

Durai V R, Roy Bhowmik S K and Mukhopadhaya B 2010 Performance evaluation of precipitation prediction skill of NCEP Global Forecasting System (GFS) over Indian region during Summer Monsoon 2008; Mausam 61(2) $139-154$.

Glahn H R and Lowry D A 1972 The Use of Model Output Statistics (MOS) in objective weather forecasting; J. Appl. Meteorol. 11(8) 1972.

Jain P, Kumar A, Maini P and Singh S V 2002 Short range SW monsoon rainfall forecasting over India using neural networks; Mausam 53(2) 225-232.

Juang H M 2014 Regional spectral model workshop in memory of John Roads and Masao Kanamitsu; Bull. Am. Meteorol. Soc., ES61-ES65 5.

Kalnay E, Kanamitsu M and Baker W E 1990 Global numerical weather prediction at the National Meteorological Center; Bull. Am. Meteorol. Soc. 71 1410-1428.

Kanamitsu M 1989 Description of the NMC global data assimilation and forecast system; Wea. Forecast 4335 342.

Kanamitsu M, Alpert J C, Campana K A, Caplan P M, Deaven D G, Iredell M, Katz B, Pan H L, Sela J and White G H 1991 Recent changes implemented into the global forecast system at NMC; Wea. Forecast 6 425-435. 
Kumar A and Maini P 1996 Statistical interpretation of general circulation model: A prospect for automation of medium range local weather forecast in India; Mausam 47(3) 227-234.

Kumar A, Mitra A K, Bohra A K, Iyengar G R and Durai V R 2012a Multi-model ensemble (MME) prediction of rainfall using neural networks during monsoon season in India; Meteorol. Appl. 19 161-169.

Kumar A, Pai D S, Singh J V, Singh R and Sikka D R 2012b Statistical models for long-range forecasting of southwest monsoon rainfall over India using step wise regression and neural network; Atmos. Clim. Sci. 2 322-336.

Maini P, Kumar A, Singh S V and Rathore L S 2002 Statistical interpretation of NWP products in India; Meteorol. Appl. 9 21-31.

Corresponding editor: A K SAHAI
Murphy A H and Katz R W 1985 Probability, statistics, and decision making in the atmospheric sciences; Westview Press, Boulder Colorado, United States, 545p.

Pankaj J, Kumar A, Maini P and Singh S V 2002 Short range SW monsoon rainfall forecasting over India using neural networks; Mausam 53(2) 225-232.

Sela J 2009 The implementation of the sigma pressure hybrid coordinates into the GFS; NCEP Office Note \#461, 25p.

Sela J 2010 The derivation of sigma pressure hybrid coordinates semi-Lagrangian model equations for the GFS; NCEP Office Note 462, 31p.

Yang F 2009 On the negative water vapor in the NCEP GFS: Sources and solution; In: 23rd conference on weather analysis and forecasting/19th conference on numerical weather prediction, 1-5 June 2009, Omaha, NE. 